\title{
Monitoring the magnetar SGR $1935+2154$ with the MAGIC telescopes
}

\author{
A. López-Oramas, ${ }^{a, b, *}$ I. Jiménez-Martínez, ${ }^{c}$ T. Hassan, ${ }^{c}$ J. Hoang,${ }^{d, e}$ S. Inoue,,${ }^{f}$ on \\ behalf of the MAGIC collaboration, F. Ambrosino, ${ }^{g}$ A. Borghese, ${ }^{h, i}$ F. Coti-Zelati, ${ }^{h, i}$ \\ J.W.T. Hessels, ${ }^{j, k}$ F. Kirsten, ${ }^{l}$ B. Marcote, ${ }^{m}$ O.S. Ould-Boukattine,${ }^{j}$ A. Papitto ${ }^{g}$ and \\ M.P. Snelders ${ }^{j}$
}

\footnotetext{
${ }^{a}$ Instituto de Astrofísica de Canarias, La Laguna, Spain

${ }^{b}$ Universidad de La Laguna, Dpto. Astrofísica, La Laguna, Spain

${ }^{c}$ Centro de Investigaciones Energéticas, Medioambientales y Tecnológicas, Madrid, Spain

${ }^{d}$ IPARCOS Institute and EMFTEL Department, Universidad Complutense de Madrid, Madrid, Spain ${ }^{e}$ now at Department of Astronomy, University of California Berkeley, Berkeley, USA

${ }^{f}$ Japanese MAGIC Group: ICRR, The University of Tokyo, Kashiwa, Japan

${ }^{g}$ INAF Osservatorio Astronomico di Roma, Roma, Italy

${ }^{h}$ Institute of Space Sciences (ICE, CSIC) Barcelona, Spain

institut d'Estudis Espacials de Catalunya (IEEC), Barcelona, Spain

${ }^{j}$ Anton Pannekoek Institute for Astronomy, University of Amsterdam, Amsterdam, The Netherlands

${ }^{k}$ ASTRON, Netherlands Institute for Radio Astronomy, Dwingeloo, The Netherlands

${ }^{l}$ Department of Space, Earth and Environment, Chalmers University of Technology Onsala Space Observatory, Sweden

m Joint Institute for VLBI ERIC, Oude Hoogeveensedijk 4, 7991 PD Dwingeloo, The Netherlands

E-mail: alicia.lopez@iac.es
}

\footnotetext{
${ }^{*}$ Presenter
} 
The Galactic magnetar SGR 1935+2154 was associated with a bright, millisecond-timescale fast radio burst (FRB) which occured in April 2020, during a flaring episode. This was the first time an FRB was unequivocally associated with a Galactic source, and the first FRB for which the nature of the emitting source was identified. Moreover, it was the first FRB with a counterpart at another wavelength correlated in time, an atypical, hard X-ray burst, which provides clear evidence for accompanying non-thermal processes. The MAGIC Telescopes are Imaging Air Cherenkov Telescopes (IACTs) sensitive to very-high-energy (VHE, E>100 GeV) gamma rays. Located at the center of the camera lies the MAGIC Central pixel, a single fully-modified photosensor-toreadout chain to measure millisecond-duration optical signals, displaying a maximum sensitivity at a wavelength of $350 \mathrm{~nm}$. This allows MAGIC to operate simultaneously both as a VHE gammaray and a fast optical telescope. The MAGIC telescopes have monitored SGR 1935+2154 in a multiwavelength campaign involving X-ray, radio and optical facilities. In this contribution, we will show the results on the search for the VHE counterpart of the first SGR-FRB source in this multiwavelength context, as well as the search for fast optical bursts with the MAGIC Central Pixel.

$37^{\text {th }}$ International Cosmic Ray Conference (ICRC 2021)

July 12th - 23rd, 2021

Online - Berlin, Germany 


\section{Introduction}

Magnetars are isolated neutron stars (NSs) with ultra-high magnetic fields ( $\mathrm{B}=10^{14}-10^{15} \mathrm{G}$ ). About thirty objects, with spin periods between 0.3 and 12 seconds, have been detected in the Galaxy, most of them during transient episodes observed only once. These magnetars can display several types of emission:

Persistent: they show bright persistent X-ray emission, powered by magnetic field decay (see [1] for a review). This persistent emission is modeled by thermal radiation from the NS hot surface (about $0.2-0.6 \mathrm{keV}$ ), reprocessed in a twisted magnetosphere through resonant cyclotron scattering. This steady component has not been detected above few keV. Searchers for steady gamma-ray emission performed at high-energy (HE, E $>100 \mathrm{MeV}$ ) by Fermi-LAT [2] set upper limits (ULs) on magnetar steady emission with the use of six years of data. Very-high-energy (VHE, E >100 GeV) observations performed by MAGIC of the magnetars 4U 0142+61 and 1E 2259+586 also resulted in non-detections, and ULs to the steady and pulsating emission were set [3]. These non-detections are in agreement with some theoretical models such as [4].

Transient: magnetars can emit flares and outbursts on different timescales, detectable in Xrays and radio. These are probably caused by large-scale rearrangements of the surface and/or magnetospheric field. They can either be accompanied or triggered by displacements of the NS crust. Magnetars can also display giant flares (GFs), which are among the most energetic $\left(10^{44}-10^{47}\right.$ $\mathrm{erg} \mathrm{s}^{-1}$ ) Galactic events. These GFs are short (lasting about a second) very-luminous hard spikes, followed by a softer pulsating tail (lasting hundreds of seconds) and a thermal afterglow (which typically lasts $<1 \mathrm{~h}$ ) seen in hard X-rays. Only four GFs have been recorded in four decades of monitoring the high-energy sky: the most recent, a GF from an extragalactic magnetar, located in the Sculptor galaxy. The Fermi-LAT satellite discovered GeV emission during this GF event [5]. This is the most energetic emission ever recorded from these events, with two photons detected above $1 \mathrm{GeV}$.

SGR $1935+2154$ is a Galactic magnetar, located at a distance of $6.6-12.5 \mathrm{kpc}[6,7]$. It is embedded in the supernova remnant SNR G57.2+0.8 and it is likely associated with a molecular cloud (MC; Zhou et al. 2020). Since its discovery in 2014 by Swift-BAT (Stamatikos et al. 2014), SGR 1935+2154 has been very active, showing several outbursts in 2015 and 2016, each time more intense in terms of the total number of bursts per active episode and in terms of the total energy emitted and in the level of the persistent emission (Younes et al. 2017; Lin et al. 2020). The most interesting and unique outburst occurred in April 28, 2020, when an extremely bright, millisecond- timescale fast radio burst (FRB), namely FRB 200428, was detected by the CHIME telescope from a direction consistent with SGR 1935+2154 [8] and confirmed by STARE2 [9] and the European dishes including the 25-m single-dish at Westerbork (the Netherlands), the $25 \mathrm{~m}$ and $20 \mathrm{~m}$ telescopes at Onsala Space Observatory (Sweden) and the $32 \mathrm{~m}$ dish in Torun (Poland) [10]. FRBs are bright transient flashes of millisecond duration, detected typically at $\mathrm{GHz}$ frequencies. FRBs were first discovered by [11] with the Parkes 64-m radio telescope. As of May 2021, a total of 147 FRB events have been detected (as reported in the FRB Community Newsletter Volume 2, Issue 05). 24 FRB repeaters have been localized and 14 host galaxies have been associated to FRB events. The FRB-event associated with SGR 1935+2154 is the only one of Galactic origin, being the rest of them originated outside of the Milky Way. Hence, SGR 1935+2154 has become the first 
source of FRBs identified with a known object and the first FRB with Galactic origin. Additionally, FRB 200428 was the first FRB accompanied by a more energetic counterpart, detected in X-rays by several instruments [12-16]. The X-ray burst had a significantly harder spectrum compared to other bursts usually observed from this and other magnetars [12].

X-ray observatories NICER [17] and Swift [18] detected different forests of bursts contemporaneously to the FRB event from SGR 1935+2154, which extended for several hours. [19] reported the detection of several millisecond-duration burst few days after the CHIME/FRB discovery, indicating the bursting phase was still active. Two days after the FRB detection, a highly-polarised, transient radio burst was reported by FAST [20].

Three more millisecond duration radio bursts were detected by CHIME/FRB on October 8, 2020 [21]. This radio emission was confirmed by FAST [22]. Several bright X-ray bursts were again detected from SGR 1935+2154 in January-February 2021 [23-25]. The several episodes of activity have been detected along almost a year.

One of the questions that intrigue the gamma-ray community is whether magnetars can emit VHE radiation. Can VHE gamma-rays be produced during GFs? Can FRB-like flares produce VHE emission? The detection of hard X-ray bursts with a non-thermal spectrum show that, at least some FRBs are able to accelerate particles and produce high-energy non-thermal emission. Also, the Fermi-LAT detection of GeV emission from an extragalactic magnetar GF demonstrates that magnetars can indeed produce high-energy gamma-ray emission. Different scenarios propose that VHE gamma-ray emission could be produced correlated with FRBs on millisecond timescales. FRBs could be produced via synchrotron maser mechanism, as proposed by [26, 27]. The emission would be triggered by strong magnetic disturbances that arise in the magnetosphere and propagate outwards, until they dissipate by interacting with the ambient nebula. $\mathrm{TeV}$ emission could be produced on millisecond (or even longer, according to [27]) timescales, but it could reach luminosity levels to be detected by IACTs. In the case of SGR 1935+2154, similar synchrotron maser shock models propose that the dissipation of disturbances within the magnetar wind is the cause of the hard X-ray burst from SGR 1935+2154 associated with FRB 200428 [28-30]. TeV emission could be produced in this case via a non-thermal tail [31].

FRBs may be potentially accompanied by "fast optical bursts" (FOBs) via different mechanisms [32]. Even if very few optical counterparts have been associated to magnetars (e. g. [33]), a very fast optical flaring episode was associated to a soft gamma repeater candidate [34]. This episode reported sub-second very-bright optical variability resembling the light-curve profiles seen during magnetar outbursts. Ra

\section{The MAGIC telescopes: gamma-ray and optical detectors}

The Major Atmospheric Gamma-ray Imaging Cherenkov (MAGIC) telescopes are a stereoscopic system composed of two $17 \mathrm{~m}$-diameter telescopes, located at $2200 \mathrm{~m}$ above sea level in the Roque de los Muchachos observatory in La Palma (Canary Islands, Spain). They detect VHE gamma-rays from as low as $50 \mathrm{GeV}$ up to $100 \mathrm{TeV}$ [35].

The MAGIC cameras are composed of 1039 highly-sensitive photomultipliers. They are able to detect the dim flashes of Cherenkov light created by gamma-initiated electromagnetic showers. The central pixel of the MAGIC camera (namely central pixel or cpix) has been adapted to measure 
millisecond-duration optical signals. It is a single fully-modified photosensor-to-readout chain, reaching a maximum sensitivity at a wavelength of $350 \mathrm{~nm}$ [36]. This allows MAGIC to operate simultaneously both as VHE gamma-ray and fast optical telescope. Since the predictions for both the most luminous FOBs associated with relatively bright FRBs and the fast optical flaring episodes seen on a SGR candidate are well within the sensitivity of the central pixel, it might be possible to detect putative emission from a FRB event from SGR 1935+2154. The central pixel has already been proven useful in the search of FRBs, as i.e., constraining the optical emission from FRB 121102 [37].

MAGIC can perform observations in two different modes. In the so-called wobble mode, the source is located off-axis from the center of the camera (typically $0.4^{\circ}$ away). In this scenario, the source position is swapped $90^{\circ}$ every 15 to 20 min of observation, allowing to simultaneously record OFF data under the same conditions that is used for background estimation. This mode is optimal for VHE observations. In the ON mode, the MAGIC telescopes track the source at the center of the camera. This allows for simultaneous observations with the central pixel. However, no simultaneous background is taken, hence dedicated OFF observations need to be scheduled or the background needs to be estimated from observations of regions in the sky with no gamma-ray detection.

\section{A multi-wavelength campaign on SGR 1935+2154}

The SGR-FRB connection established with the detection of FRB 200428, have made SGR $1935+2154$ a highly interesting source to be studied. Considering the uniqueness of the 2020 event, we started a multi-wavelength (MWL) campaign to monitor the status of the magnetar and potentially detect either gamma or FOB emission for the first time. Unfortunately, the April 2020 event took place during the COVID19 lockdown, when MAGIC was under shutdown. During the following months, we organized coordinated MWL observations with radio (Westerbork and Onsala), optical (SiFAP2 camera [38] mounted on the Telescopio Nazionale Galileo; TNG) and $\mathrm{X}$-ray (Swift) facilities to monitor the behavior of this magnetar while it was visible from the MAGIC site. This campaign took place between July and October 2020. The second detection of millisecond radio emission by CHIME six months after the first event, confirmed that SGR 1935+2154 was still active and that FRB-like emission was still possible any time.

The strategy was to monitor the source at least twice per month and trigger additional observations whenever flaring emission or outbursts were detected in other frequencies (namely X-rays or radio). The list of performed coordinated observations is collected in Table 1.

No VHE counterpart has been identified in a daily basis in MAGIC data. Likewise, the overall VHE emission is not significant. We have calculated upper limits (ULs at $95 \%$ confidence level) to the flux and the corresponding lightcurve above $100 \mathrm{GeV}$. Similarly, no radio bursts were reported by Westerbork or Onsala. Optical TNG/SiFAP2 observations could not identify any optical burst either. On the contrary, Swift reported several burst episodes on different nights (Borghese et al. in prep).

MAGIC observations were scheduled both in wobbling and ON modes, to increase VHE sensitivity and allow simultaneous central-pixel observations, respectively. Simultaneous gammaray/optical cpix observations started to be taken from October 9, 2020, one night after the new 
millisecond radio detections reported by CHIME. Also on October 9, FAST reported the presence of pulsed-emission and several radio bursts [22]. Simultaneous MAGIC and Swift observations were scheduled via ToO for that night. A forest of bursts was detected in Swift (Borghese et al. in prep). The analysis does not reveal any persistent VHE emission from MAGIC data. A detailed analysis is ongoing for the identification of burst-like signals within both the short-timescale VHE and optical regimes. The Onsala radio telescope observed later on that night for $50 \mathrm{~min}$, but no bursting activity was detected.

\begin{tabular}{|c|c|c|c|c|}
\hline $\begin{array}{c}\text { Date } \\
\text { (MJD) }\end{array}$ & $\begin{array}{c}\text { MAGIC } \\
\text { (gamma/optical cpix) }\end{array}$ & $\begin{array}{c}\text { Swift } \\
\text { (X-rays) }\end{array}$ & $\begin{array}{c}\text { TNG/SiFAP2 } \\
\text { (optical) }\end{array}$ & $\begin{array}{c}\text { Westerbork/Onsala } \\
\text { (radio) }\end{array}$ \\
\hline $59053.96-59054.04$ & yes/no & yes & no & yes/no \\
$59080.88-59080.97$ & yes/no & no & no & no/no \\
$59102.95-59103.03$ & yes/no & no & yes & yes/no \\
$59109.95-59110.03$ & yes/no & yes & no & yes/no \\
$59110.86-59110.94$ & yes/no & yes & no & yes/no \\
$59111.85-59111.93$ & yes/no & yes & no & yes/no \\
$59131.89-59131.98$ & yes/yes & yes & no & no/yes \\
\hline
\end{tabular}

Table 1: MWL observations of SGR 1935+2154 .

\section{Conclusions}

SGR $1935+2154$ is the first source identified as a FRB emitter of a known nature. In addition, it is the source associated to the first FRBs with detected multi-wavelength counterparts, and it is located within the Milky Way. Due to these extremely unique characteristics, we have started a monitoring campaign to follow-up its behavior in a MWL context. No VHE emission has been detected yet with MAGIC. A detailed analysis for the search of burst-like VHE or FOB events is still ongoing. Coordinated multi-frequency observations of SGR 1935+2154 will help us better understand magnetars and FRBs. A positive detection either in the VHE or optical regimes would be a paramount discovery for both the Galactic transients and VHE fields.

\section{Acknowledgements}

We acknowledge the support from the agencies and organizations listed here: https://magic.mpp.mpg.de/acknowledgments_ICRC2021/

\section{References}

[1] S. Mereghetti, Astronomy and Astrophysics Reviews 15, 225 (2008), 0804.0250.

[2] J. Li, N. Rea, D. Torres, and E. de Oña-Wilhelmi, 835, 30 (2017), 1607.03778.

[3] J. Aleksić, L. A. Antonelli, P. Antoranz, et al., 549, A23 (2013), 1211.1173. 
[4] D. Viganò, D. F. Torres, and J. Martín, 453, 2599 (2015), 1507.04021.

[5] M. Ajello et al. (Fermi-LAT), Nature Astronomy 5, 385-39 (2021).

[6] R. Kothes, X. Sun, B. Gaensler, and W. Reich, 852, 54 (2018), 1711.11146.

[7] P. Zhou, X. Zhou, Y. Chen, et al., 905, 99 (2020), 2005.03517.

[8] B. C. Andersen et al. (CHIME/FRB Collaboration), 587, 54 (2020), 2005 . 10324.

[9] C. D. Bochenek, V. Ravi, K. V. Belov, et al., 587, 59 (2020), 2005. 10828.

[10] F. Kirsten, M. P. Snelders, M. Jenkins, et al., Nature Astronomy 5, 414 (2021), 2007.05101.

[11] D. R. Lorimer, M. Bailes, McLaughlin, et al., Science 318, 777 (2007), 0709 . 4301.

[12] S. Mereghetti, V. Savchenko, C. Ferrigno, et al., Astrophysical Journal 898, L29 (2020), 2005.06335.

[13] M. Tavani, C. Casentini, A. Ursi, et al., Nature Astronomy 5, 401 (2021), 2005.12164.

[14] A. Ridnaia, D. Svinkin, D. Frederiks, et al., Nature Astronomy 5, 372 (2021), 2005 . 11178.

[15] G. Younes, M. G. Baring, C. Kouveliotou, et al., Nature Astronomy (2021), 2006.11358.

[16] C. Li, L. Lin, S. Sion, et al., Nature Astronomy 5, 378-384 (2021).

[17] G. Younes, T. Guver, T. Enoto, et al., The Astronomer's Telegram 13678, 1 (2020).

[18] D. M. Palmer, The Astronomer's Telegram 13675, 1 (2020).

[19] A. Borghese, N. Rea, F. Coti Zelati, et al., The Astronomer's Telegram 13720, 1 (2020).

[20] S. N. Zhang, Y. L. Tuo, S. L. Xiong, et al., The Astronomer's Telegram 13687, 1 (2020).

[21] D. Good and CHIME/FRB Collaboration, The Astronomer's Telegram 14074, 1 (2020).

[22] W. Zhu, B. Wang, D. Zhou, et al., The Astronomer's Telegram 14084, 1 (2020).

[23] Y. Huang, S. J. Zheng, F. J. Lu, et al. (Gecam Team), GRB Coordinates Network 29363, 1 (2021).

[24] O. J. Roberts, J. Wood, R. Hamburg, A. von Kienlin, P. Veres, and G. Younes, The Astronomer's Telegram 14359, 1 (2021).

[25] A. Borghese, F. Coti Zelati, N. Rea, P. Esposito, and G. L. Israel, The Astronomer's Telegram 14388, 1 (2021).

[26] Y. Lyubarsky, 442, L9 (2014), 1401.6674.

[27] K. Murase, K. Kashiyama, and P. Mészáros, 461, 1498 (2016), 1603.08875.

[28] B. Margalit, P. Beniamini, N. Sridhar, and B. Metzger, 899, L27 (2020), 2005.05283. 
[29] Q. Wu, G. Q. Zhang, F. Y. Wang, and Z. G. Dai, 900, L26 (2020), 2008.05635.

[30] S. Yamasaki, K. Kashiyama, and K. Murase, arXiv e-prints arXiv:2008.03634 (2020), 2008. 03634.

[31] B. D. Metzger, K. Fang, and B. Margalit, 902, L22 (2020), 2008. 12318.

[32] Y. Yang, B. Zhang, and J. Wei, 878, 89 (2019), 1905.02429.

[33] V. S. Dhillon, T. R. Marsh, F. Hulleman, et al., 363, 609 (2005), astro-ph/0508039.

[34] A. Stefanescu, G. Kanbach, A. Słowikowska, et al., Nature 455, 503 (2008), 0809 . 4043.

[35] J. Aleksić et al. (MAGIC), Astroparticle Physics 72, 76 (2016), 1409. 5594.

[36] T. Hassan, J. Hoang, M. López Moya, et al., in 35th International Cosmic Ray Conference (ICRC2017) (2017), vol. 301, p. 807, 1708.07698.

[37] V. A. Acciari, S. Ansoldi, L. A. Antonelli, et al. (MAGIC Collaboration), 481, 2479 (2018), 1809.00663.

[38] A. Ghedina, F. Leone, F. Ambrosino, et al., in Ground-based and Airborne Instrumentation for Astronomy VII (2018), vol. 10702 of Society of Photo-Optical Instrumentation Engineers (SPIE) Conference Series, p. 107025Q.

\section{The MAGIC Collaboration}

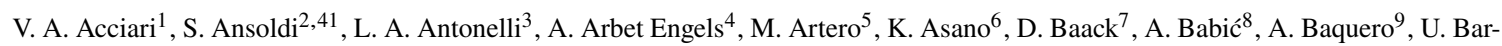
res de Almeida ${ }^{10}$, J. A. Barrio ${ }^{9}$, I. Batković ${ }^{11}$, J. Becerra González ${ }^{1}$, W. Bednarek ${ }^{12}$, L. Bellizzi ${ }^{13}$, E. Bernardini ${ }^{14}$, M. Bernardos ${ }^{11}$, A. Berti ${ }^{15}$, J. Besenrieder ${ }^{15}$, W. Bhattacharyya ${ }^{14}$, C. Bigongiari ${ }^{3}$, A. Biland ${ }^{4}$, O. Blanch ${ }^{5}$, H. Bökenkamp ${ }^{7}$, G. Bonnoli ${ }^{16}$, Ž. Bošnjak ${ }^{8}$, G. Busetto ${ }^{11}$, R. Carosi ${ }^{17}$, G. Ceribella ${ }^{15}$, M. Cerruti ${ }^{18}$, Y. Chai ${ }^{15}$, A. Chilingarian ${ }^{19}$, S. Cikota ${ }^{8}$, S. M. Colak ${ }^{5}$, E. Colombo ${ }^{1}$, J. L. Contreras ${ }^{9}$, J. Cortina ${ }^{20}$, S. Covino ${ }^{3}$, G. D’Amico ${ }^{15,42}$, V. D’Elia ${ }^{3}$, P. Da Vela ${ }^{17,43}$, F. Dazzi ${ }^{3}$, A. De Angelis ${ }^{11}$, B. De Lotto ${ }^{2}$, M. Delfino ${ }^{5,44}$, J. Delgado ${ }^{5,44}$, C. Delgado Mendez $^{20}$, D. Depaoli ${ }^{21}$, F. Di Pierro ${ }^{21}$, L. Di Venere ${ }^{22}$, E. Do Souto Espiñeira ${ }^{5}$, D. Dominis Prester ${ }^{23}$, A. Donini ${ }^{2}$, D. Dorner ${ }^{24}$, M. Doro ${ }^{11}$, D. Elsaesser ${ }^{7}$, V. Fallah Ramazani ${ }^{25,45}$, A. Fattorini ${ }^{7}$, M. V. Fonseca ${ }^{9}$, L. Font ${ }^{26}$, C. Fruck ${ }^{15}$, S. Fukami ${ }^{6}$, Y. Fukazawa ${ }^{27}$, R. J. García López ${ }^{1}$, M. Garczarczyk ${ }^{14}$, S. Gasparyan ${ }^{28}$, M. Gaug ${ }^{26}$, N. Giglietto ${ }^{22}$, F. Giordano ${ }^{22}$, P. Gliwny ${ }^{12}$, N. Godinović ${ }^{29}$, J. G. Green ${ }^{3}$, D. Green ${ }^{15}$, D. Hadasch ${ }^{6}$, A. Hahn ${ }^{15}$, L. Heckmann ${ }^{15}$, J. Herrera ${ }^{1}$, J. Hoang ${ }^{9,46}$, D. Hrupec ${ }^{30}$, M. Hütten ${ }^{15}$, T. Inada ${ }^{6}$, K. Ishio ${ }^{12}$, Y. Iwamura ${ }^{6}$, I. Jiménez Martínez ${ }^{20}$, J. Jormanainen ${ }^{25}$, L. Jouvin ${ }^{5}$, M. Karjalainen ${ }^{1}$, D. Kerszberg ${ }^{5}$, Y. Kobayashi ${ }^{6}$, H. Kubo ${ }^{31}$, J. Kushida ${ }^{32}$, A. Lamastra ${ }^{3}$, D. Lelas ${ }^{29}$, F. Leone ${ }^{3}$, E. Lindfors ${ }^{25}$, L. Linhoff $^{7}$, S. Lombardi ${ }^{3}$, F. Longo ${ }^{2,47}$, R. López-Coto ${ }^{11}$, M. López-Moya ${ }^{9}$, A. López-Oramas ${ }^{1}$, S. Loporchio ${ }^{22}$, B. Machado de Oliveira Fraga $^{10}$, C. Maggio ${ }^{26}$, P. Majumdar ${ }^{33}$, M. Makariev ${ }^{34}$, M. Mallamaci ${ }^{11}$, G. Maneva ${ }^{34}$, M. Manganaro ${ }^{23}$, K. Mannheim ${ }^{24}$, L. Maraschi ${ }^{3}$, M. Mariotti ${ }^{11}$, M. Martínez ${ }^{5}$, D. Mazin ${ }^{6,15}$, S. Menchiari ${ }^{13}$, S. Mender ${ }^{7}$, S. Mićanović2 ${ }^{23}$, D. Miceli ${ }^{2,49}$, T. Miener ${ }^{9}$, J. M. Miranda ${ }^{13}$, R. Mirzoyan ${ }^{15}$, E. Molina ${ }^{18}$, A. Moralejo ${ }^{5}$, D. Morcuende ${ }^{9}$, V. Moreno ${ }^{26}$, E. Moretti ${ }^{5}$, T. Nakamori ${ }^{35}$, L. Nava ${ }^{3}$, V. Neustroev ${ }^{36}$, C. Nigro ${ }^{5}$, K. Nilsson ${ }^{25}$, K. Nishijima ${ }^{32}$, K. Noda ${ }^{6}$, S. Nozaki ${ }^{31}$, Y. Ohtani ${ }^{6}$, T. Oka ${ }^{31}$, J. Otero-Santos ${ }^{1}$, S. Paiano ${ }^{3}$, M. Palatiello ${ }^{2}$, D. Paneque ${ }^{15}$, R. Paoletti ${ }^{13}$, J. M. Paredes ${ }^{18}$, L. Pavletić ${ }^{23}$, P. Peñil ${ }^{9}$, M. Persic ${ }^{2,50}$, M. Pihet ${ }^{15}$, P. G. Prada Moroni ${ }^{17}$, E. Prandini ${ }^{11}$, C. Priyadarshi ${ }^{5}$, I. Puljak ${ }^{29}$, W. Rhode ${ }^{7}$, M. Ribó ${ }^{18}$, J. Rico ${ }^{5}$, C. Righi ${ }^{3}$, A. Rugliancich ${ }^{17}$, N. Sahakyan ${ }^{28}$, T. Saito ${ }^{6}$, S. Sakurai ${ }^{6}$, K. Satalecka ${ }^{14}$, F. G. Saturni ${ }^{3}$, B. Schleicher ${ }^{24}$, K. Schmidt $^{7}$, T. Schweizer ${ }^{15}$, J. Sitarek ${ }^{12}$, I. Šnidaric ${ }^{37}$, D. Sobczynska ${ }^{12}$, A. Spolon ${ }^{11}$, A. Stamerra ${ }^{3}$, J. Strišković ${ }^{30}$, D. Strom ${ }^{15}$, M. Strzys ${ }^{6}$, Y. Suda ${ }^{27}$, T. Suric ${ }^{37}$, M. Takahashi ${ }^{6}$, R. Takeishi' ${ }^{6}$, F. Tavecchio ${ }^{3}$, P. Temnikov ${ }^{34}$, T. Terzić ${ }^{23}$, M. Teshima ${ }^{15,6}$, L. Tosti ${ }^{38}$, S. Truzzi ${ }^{13}$, A. Tutone ${ }^{3}$, S. Ubach ${ }^{26}$, J. van Scherpenberg ${ }^{15}$, G. Vanzo ${ }^{1}$, M. Vazquez Acosta ${ }^{1}$, S. Ventura ${ }^{13}$, V. Verguilov ${ }^{34}$, C. F. Vigorito ${ }^{21}$, V. Vitale ${ }^{39}$, I. Vovk $^{6}$, M. Will ${ }^{15}$, C. Wunderlich ${ }^{13}$, T. Yamamoto $^{40}$, and D. Zarić ${ }^{29}$

\footnotetext{
${ }^{1}$ Instituto de Astrofísica de Canarias and Dpto. de Astrofísica, Universidad de La Laguna, E-38200, La Laguna, Tenerife, Spain ${ }^{2}$ Università di Udine and INFN Trieste, I-33100 Udine, Italy ${ }^{3}$ National Institute for Astrophysics (INAF), I-00136 Rome, Italy ${ }^{4}$ ETH Zürich, CH-8093 Zürich, Switzerland ${ }^{5}$ Institut de Física d'Altes Energies (IFAE), The Barcelona Institute of Science and Technology
} 
(BIST), E-08193 Bellaterra (Barcelona), Spain ${ }^{6}$ Japanese MAGIC Group: Institute for Cosmic Ray Research (ICRR), The University of Tokyo, Kashiwa, 277-8582 Chiba, Japan ${ }^{7}$ Technische Universität Dortmund, D-44221 Dortmund, Germany ${ }^{8}$ Croatian MAGIC Group: University of Zagreb, Faculty of Electrical Engineering and Computing (FER), 10000 Zagreb, Croatia ${ }^{9}$ IPARCOS Institute and EMFTEL Department, Universidad Complutense de Madrid, E-28040 Madrid, Spain ${ }^{10}$ Centro Brasileiro de Pesquisas Físicas (CBPF), 22290-180 URCA, Rio de Janeiro (RJ), Brazil ${ }^{11}$ Università di Padova and INFN, I-35131 Padova, Italy ${ }^{12}$ University of Lodz, Faculty of Physics and Applied Informatics, Department of Astrophysics, 90-236 Lodz, Poland ${ }^{13}$ Università di Siena and INFN Pisa, I-53100 Siena, Italy ${ }^{14}$ Deutsches Elektronen-Synchrotron (DESY), D-15738 Zeuthen, Germany ${ }^{15}$ Max-Planck-Institut für Physik, D-80805 München, Germany ${ }^{16}$ Instituto de Astrofísica de Andalucía-CSIC, Glorieta de la Astronomía s/n, 18008, Granada, Spain ${ }^{17}$ Università di Pisa and INFN Pisa, I-56126 Pisa, Italy ${ }^{18}$ Universitat de Barcelona, ICCUB, IEEC-UB, E-08028 Barcelona, Spain ${ }^{19}$ Armenian MAGIC Group: A. Alikhanyan National Science Laboratory, 0036 Yerevan, Armenia ${ }^{20}$ Centro de Investigaciones Energéticas, Medioambientales y Tecnológicas, E-28040 Madrid, Spain ${ }^{21}$ INFN MAGIC Group: INFN Sezione di Torino and Università degli Studi di Torino, I-10125 Torino, Italy 22 INFN MAGIC Group: INFN Sezione di Bari and Dipartimento Interateneo di Fisica dell'Università e del Politecnico di Bari, I-70125 Bari, Italy ${ }^{23}$ Croatian MAGIC Group: University of Rijeka, Department of Physics, 51000 Rijeka, Croatia ${ }^{24}$ Universität Würzburg, D-97074 Würzburg, Germany ${ }^{25}$ Finnish MAGIC Group: Finnish Centre for Astronomy with ESO, University of Turku, FI-20014 Turku, Finland ${ }^{26}$ Departament de Física, and CERES-IEEC, Universitat Autònoma de Barcelona, E-08193 Bellaterra, Spain ${ }^{27}$ Japanese MAGIC Group: Physics Program, Graduate School of Advanced Science and Engineering, Hiroshima University, 739-8526 Hiroshima, Japan ${ }^{28}$ Armenian MAGIC Group: ICRANet-Armenia at NAS RA, 0019 Yerevan, Armenia ${ }^{29}$ Croatian MAGIC Group: University of Split, Faculty of Electrical Engineering, Mechanical Engineering and Naval Architecture (FESB), 21000 Split, Croatia ${ }^{30}$ Croatian MAGIC Group: Josip Juraj Strossmayer University of Osijek, Department of Physics, 31000 Osijek, Croatia ${ }^{31}$ Japanese MAGIC Group: Department of Physics, Kyoto University, 606-8502 Kyoto, Japan ${ }^{32}$ Japanese MAGIC Group: Department of Physics, Tokai University, Hiratsuka, 259-1292 Kanagawa, Japan ${ }^{33}$ Saha Institute of Nuclear Physics, HBNI, 1/AF Bidhannagar, Salt Lake, Sector-1, Kolkata 700064, India ${ }^{34}$ Inst. for Nucl. Research and Nucl. Energy, Bulgarian Academy of Sciences, BG-1784 Sofia, Bulgaria ${ }^{35}$ Japanese MAGIC Group: Department of Physics, Yamagata University, Yamagata 990-8560, Japan ${ }^{36}$ Finnish MAGIC Group: Astronomy Research Unit, University of Oulu, FI-90014 Oulu, Finland ${ }^{37}$ Croatian MAGIC Group: Ruđer Bošković Institute, 10000 Zagreb, Croatia ${ }^{38}$ INFN MAGIC Group: INFN Sezione di Perugia, I-06123 Perugia, Italy ${ }^{39}$ INFN MAGIC Group: INFN Roma Tor Vergata, I-00133 Roma, Italy ${ }^{40}$ Japanese MAGIC Group: Department of Physics, Konan University, Kobe, Hyogo 6588501, Japan ${ }^{41}$ also at International Center for Relativistic Astrophysics (ICRA), Rome, Italy ${ }^{42}$ now at Department for Physics and Technology, University of Bergen, NO-5020, Norway ${ }^{43}$ now at University of Innsbruck ${ }^{44}$ also at Port d'Informació Científica (PIC), E-08193 Bellaterra (Barcelona), Spain ${ }^{45}$ now at Ruhr-Universität Bochum, Fakultät für Physik und Astronomie, Astronomisches Institut (AIRUB), 44801 Bochum, Germany ${ }^{46}$ now at Department of Astronomy, University of California Berkeley, Berkeley CA 9472047 also at Dipartimento di Fisica, Università di Trieste, I-34127 Trieste, Italy ${ }^{49}$ now at Laboratoire d'Annecy de Physique des Particules (LAPP), CNRS-IN2P3, 74941 Annecy Cedex, France ${ }^{50}$ also at INAF Trieste and Dept. of Physics and Astronomy, University of Bologna, Bologna, Italy 